\title{
Erratum to: Kurt Hellmann D.M., D.Phil. Oxon. A happy 90th birthday!
}

\author{
Suzanne A. Eccles $\cdot$ Rudolf K. Steiner
}

Received: 14 November 2014/ Accepted: 6 February 2015/Published online: 8 August 2012

(C) Springer Science+Business Media B.V. 2012

\section{Erratum to: Clin Exp Metastasis}

DOI 10.1007/s10585-012-9490-4

Due to a technical problem arising during the correction stage of the above-cited dedication, several errors appeared in the online and printed version. To put matters right, we republish the corrected article on the following pages.
We sincerely apologize to both authors for any inconvenience caused by the mistakes that appeared in the first published version.

The online version of the original article can be found under doi: 10.1007/s10585-012-9490-4.

\footnotetext{
S. A. Eccles $(\square)$

Tumour Biology and Metastasis, Cancer Research UK Cancer

Therapeutics Unit, The Institute of Cancer Research, Sutton,

Surrey, UK

e-mail: sue.eccles@icr.ac.uk

Present Address:

R. K. Steiner

Former EORTC \& ICRF Clinical Research Fellow, Bionstrasse

15, 8006 Zurich, Switzerland
} 


\title{
Kurt Hellmann D.M., D.Phil. Oxon. A happy 90th birthday!
}

\author{
Suzanne A. Eccles · Rudolf K. Steiner
}

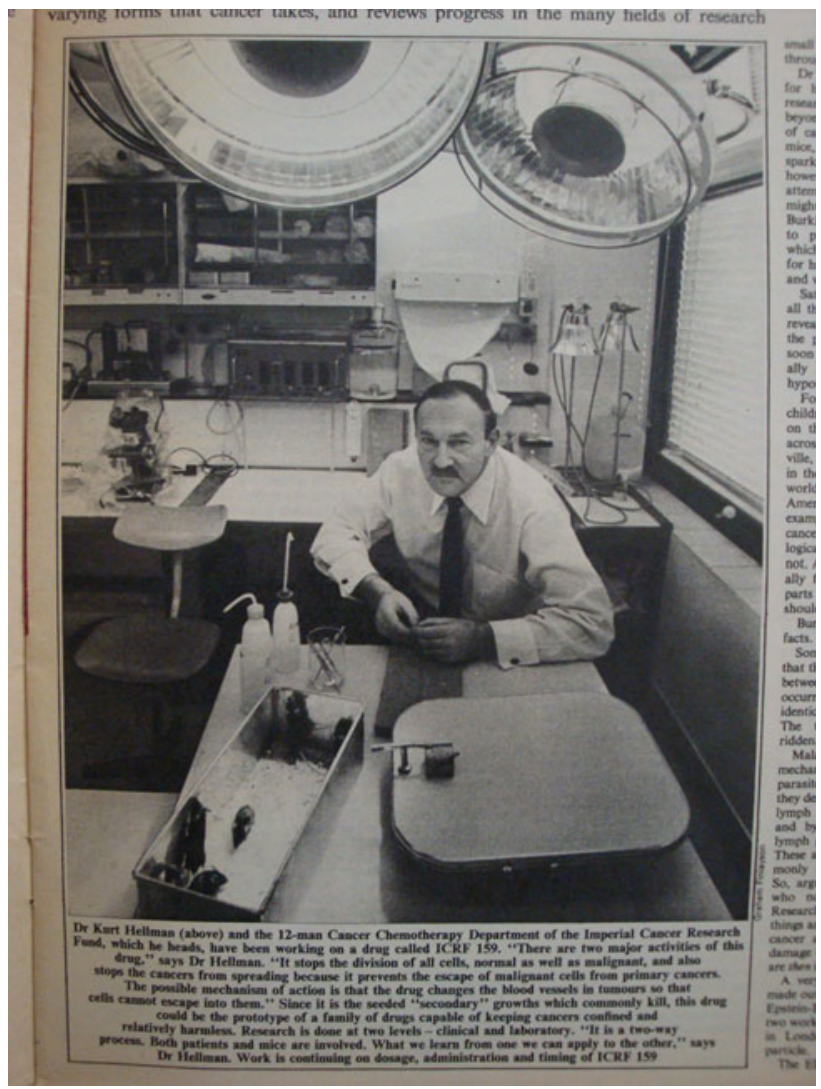

May 12th marked the 90th birthday of the medical pharmacologist Kurt Hellmann-one of the most eminent pioneers in metastasis research-whose foresight in science and medicine is unparalleled.

Inspired research and a close eye for detail led Kurt to the discovery of the unique cytostatic, cytoprotective and antimetastatic biological activities of bisdioxopiperazines in his laboratory at the ICRF Cancer Chemotherapy Unit Lincoln's Inn Fields, London, in the early 1970s. He resurrected the EDTA-analogues synthesised by Andrew Creighton but they had been patented by Geigy AG (now Novartis). Their characteristics and chemistry are excellently described by Marie Bakowski in Cancer Treatment Reviews 3, 95-107, 1976.

The selective antimetastatic property of razoxane [ICRF 159] was first observed in the Lewis lung carcinoma (3LL) model utilized by Kurt as a screening system for studying compounds with potential inhibitory activity on metastasis. This work was regarded as a major breakthrough when published with Karen Burrage in Nature on 18 October 1969. A follow-up study by Kurt and his co-worker A.W. LeServe was published in the British Medical Journal in early 1972 ('Metastases and the normalization of tumour blood vessels by ICRF 159: a new type of drug action') and reprinted as a 'classic' paper with an accompanying Editorial in Clinical \& Experimental Metastasis in 2008.

This important paper clearly showed for the first time that it was possible to 'normalize' tumour-induced pathologic vasculature by the prevention of intra-tumoral haemorrhage and hence the spread of cancer cells and subsequent lethal metastasis. This observation pre-dates by decades the recent popularisation of the idea that reversion of tumour vasculature to a more normal morphology could provide a valid therapeutic approach.

In the clinical context, the orally active razoxane analogue was subsequently shown by the British surgeon Jonathan Gilbert to prevent liver metastases in the adjuvant treatment of colorectal cancer. More recently it has been demonstrated by the Austrian radiotherapist Walter Rhomberg to potentiate certain schedules of chemoradiotherapy and to suppress metastasis in soft tissue sarcomas, in part by improvement of oxygen and drug delivery.

In the early 1970s, Kurt Hellmann brought razoxane to the attention of Abraham Goldin and Eugene Herman in the Laboratory of Chemical Pharmacology at the Clinical Center of the National Cancer Institute, Bethesda, MD. Herman found this intracellular active chelator to be highly effective in reducing anthracycline-induced cardiotoxicity in all species tested. This led to rapid NCI-supported clinical development of the parenteral, highly water soluble pure enantiomer $(+)$ 
dexrazoxane [ICRF 187] by James Speyer (published in 1988/92) and definitively by Sandra Swain (published in 1997/98). These pivotal US prospective clinical studies clearly demonstrated effective, long-term cardioprotection by dexrazoxane, preserving the myocardial reserves in breast cancer patients but with no additional toxicities. These findings were subsequently confirmed in 2006 in a French registration trial led by Michel Marty. Currently, dexrazoxane is the only FDA/EMA approved agent for preventing anthracycline-induced cardiotoxicity which according to Ewer \& Speyer 'must become an essential part of anthracycline-based modern chemotherapy practice' allowing for the optimization of such treatment modalities.

In the October 2010 issue of Lancet Oncology, the paediatric cardiologist Steven Lipshultz at the Department of Pediatrics, University of Miami Miller School of Medicine and the pediatric oncologist Stephen Sallan at Dana-Farber/ Children's Hospital Cancer Center, showed in a landmark prospective, randomised, multicentre trial of doxorubicintreated children with high-risk acute lymphoblastic leukaemia (ALL) that dexrazoxane provides cardioprotection without compromising oncological efficacy or enhancing the rate of second malignancies in the long-term follow-up. On April 1, 2012 a further report by the same group published in the Journal of Clinical Oncology underscored the importance of dexrazoxane administered before each dose of doxorubicin to reduce cardiotoxicity and urged the use of a comprehensive panel of biomarkers to assess cardiac status in the long-term follow-up of patients treated with the clinically proven, cost-effective anthracyclines.

This account cannot end without quoting verbatim from a letter of support from James Doroshow, Director, Division of Cancer Treatment and Diagnosis US National Cancer Institute, for a meeting organised by Kurt Hellmann on 'Cancer Therapy Improvement by Toxicity Reduction and Cardioprotection' at the Royal Society of Medicine, London, in October 2006. Doroshow wrote "it is a great pleasure to extend to you and the extraordinary group of experts in the area of anthracycline clinical pharmacology that you have assembled, my very best wishes for what will be an important examination of the continuing role of dexrazoxane in the prevention of cardiac damage produced by the anthracycline antibiotics. The critical roles played by all of the speakers in the Symposium in developing our current understanding of the mechanism of anthracycline cardiac toxicity were essential in the approval of dexrazoxane by the US Food and Drug Administration. With a decade of clinical application in hand, it would seem that an expansion of the use of this important protective agent should be considered...".

Further exploration of the drug's cytoprotective activity beyond the FDA/EMA approved treatment showed that the serious consequences of accidental anthracycline extravasation could also be ameliorated or prevented by dexrazoxane. This work was undertaken in 2007 because of the widespread chelation activity of the bisdioxopiperazines and the known inhibition of cell division at the G2/M transition. Additional considerations were the ability of both piperazines to block topoisomerase II alpha, cellular senescence, apoptosis and induction of thrombospondin-1. The neuroprotective dexrazoxane was found to enhance the efficacy of mitoxantrone in a rat model of multiple sclerosis by the German neurologist Franz Weilbach.

In addition, razoxane and dexrazoxane were recently shown to reduce beta amyloid peptide levels in preclinical studies of Alzheimer's disease by Nigel Greig at NIH, confirming Gerhard Zbinden's findings in 1983 that protection is possible in neuronal tissues. A recently pubished monograph edited by Hellmann \& Rhomberg bears witness to the remarkable experimental and clinical adventure of 'Razoxane and Dexrazoxane-Two Multifunctional Agents' as experienced by some of its key investigators (Springer 2011, ISBN: 987-90-481-9167-3). Thus, initial laboratory-based studies in mouse models of metastasis have yielded clinical benefits and mechanistic insights into drug effects on tumours and normal tissues far beyond anything envisaged in those pioneering days at Lincoln's Inn Fields.

Kurt Hellmann was born in Nürnberg, Bavaria, where he attended primary school from 1927 to 1932 and enjoyed the first year of the 'Reformgymnasium' before having to emigrate as a 10 year old boy with his parents and his elder brother to England in March 1933. After finishing school in London he served during wartime as an engineer. After the war he studied chemistry in London. Between 1950 and 1957 he continued his academic career at the Department of Anatomy in Oxford and concurrently at the Physiological Institute of the Royal Navy in Oxford and Singapore. He earned a Ph.D. in Pharmacology (1953), a BM ChB in Medicine (1958) and a D.M. degree (1964) at Magdalen \& Balliol College Oxford where he won the prestigious Radcliffe Prize for Medical Research for his histochemical investigations on cholinesterase and amine oxidase in the skin (J Physiol, 129, 454-463, 1955). After clinical training at the Radcliffe Infirmary, Oxford (1958-60) he joined Reckitt \& Sons, Hull, to lead its Department of Pharmacology but was seconded to undertake medical research at the Department of Pharmacology, Royal College of Surgeons, London. In 1962 he became Director of the newly formed Department of Cancer Chemotherapy at the Imperial Cancer Research Fund London (ICRF; now the Cancer Research UK London Research Institute) - a post which he held until 1987 alongside clinical activities as an Honorary Consultant \& Visiting Professor at the Radiotherapy \& Oncology Department, Westminster Hospital, London (now Imperial College) from 1972 to 1993.

In March 1974, Kurt Hellmann founded with his coeditor Stephen Carter (Director of the Division of Cancer 
Treatment, US National Cancer Institute) the highly regarded journal 'Cancer Treatment Reviews' which he edited from volume 1(1), 1974 to volume 18(4) in December 1991. The purpose of 'Cancer Treatment Reviews' was to analyse emerging data authoritatively and in a thought-provoking style in order to stimulate further advances in research and treatment. What a workload apart from his daily commitments in the laboratory and clinic!

His long-standing association with Stephen Carter and his clinical research fellow Marie Bakowski culminated in publication of the widely read 'Chemotherapy of Cancer' in 1977 as a convenient reference and guidebook for every practising medical oncologist. In March 1974 he organized the first meeting of the E.O.R.T.C. Metastasis Club which he created together with Silvio Garattini, the Director of the Mario Negri Institute for Pharmacological Research, Milan. Some 30 members from France, Germany, Italy, The Netherlands, Switzerland and the UK gathered under his presidency at ICRF to discuss intravascular events in cancer dissemination. In the early 1980's the ever-expanding interest in the field of metastasis encouraged the development of this informal club into the 'Metastasis Research Society' with Garth Nicolson and Suzanne Eccles amongst others. Its first international meeting was organised by Kurt and Sue at the Strand Palace Hotel, London, in October 1984, shortly after inauguration of 'Clinical \& Experimental Metastasis' (this soon became the Society's official journal) which he co-edited with Garth Nicholson, Sue Eccles, Tatsuro Irimura and (Associate Editor) Luka Milas until the 1998. He is now a valued Emeritus Editor and still takes a keen interest in the journal.

It is a testament to Kurt Hellmann's initiatives and foresight that both the Society and journal continue to thrive. The Metastasis Research Society has attracted over 300 members from 30 countries and its biennial international conferences (held in most major European countries, the USA and Japan) routinely attract 300-400 delegates from all over the world. The next conference will be held in Brisbane in September 2012, its first time in Australia. Clinical \& Experimental Metastasis enjoys a reputation as a high profile specialist journal with an impact factor fast approaching 5.0; serving both research scientists and clinicans with interests in the basic biology, prevention and treatment of disseminated disease.

In 1972 Kurt Hellmann acted as Chairman of the British Association of Cancer Research (BACR) and gave the 'Erasmus Wilson Lecture' of the Royal College of Surgeons. When the Queen opened a new wing at ICRF's Lincoln's Inn site in 1973 he was given the task of showing her around the Department of Cancer Chemotherapy in which she took great interest. Some 10 years later, as President of the Oncology Section of the Royal Society of Medicine, UK he was invited to give the 'Haddow Lecture' of BACR.
Kurt Hellmann also made an memorable debut in 'A Career in Pharmacology' in 1961 filmed by members of staff of the Department of Pharmacology at the Royal College of Surgeons of England, then based at Examination Hall, Queen Square London, co-starring with the later Nobel Prize winner John Vane and others (Wellcome Trust, re-edited DVD, 2009). Some may recall a unforgettable showing of this film at the MRS 2000 conference dinner at the Royal College of Surgeons in London. The Wellcome Library also holds a recording of a 1993 interview between Kurt Hellmann and two other colleagues with Sir Stanford Cade (1895-1973). Cade was a pioneer in radiotherapy at the Westminster Hospital and an Air Vice Marshall of the Royal Airforcewhose invaluable collection of case summaries was rescued by Hellmann upon the closure of the Westminster Hospital and given to the Contemporary Medical Archives Centre of the Wellcome Trust's Library (GC147). The published proceedings of 'The Stanford Cade Symposium' organized by Kurt Hellmann at the Royal Institution, London, in 1973 bear witness to his early interests in preserving our medical heritage. Kurt Hellmann's advice to all of us engaged in struggling to make progress along the twisted yellow brick road of modern drug discovery and development may best be summarized with his characteristic chuckle: "you just have to live long enough...!"

Kurt Hellmann continues to be active as a writer, advisor, critical discussant and benefactor of pharmacological drug research and education, supported by his wife Jane, a former doctor with the blood transfusion services and a talented gardener.

Suzanne A. Eccles, PhD, Tumour Biology and Metastasis, Cancer Research UK Cancer Therapeutics Unit at The Institute of Cancer Research, Sutton, Surrey.

Rudolf Steiner, M.D., Zurich, Switzerland, former EORTC \& ICRF clinical research fellow at ICRF's extramural Breast Unit, Guy's Hospital, at the MRC Leukaemia Unit, Hammersmith Hospital, \& at the Tissue Relationships Laboratory at ICRF, Lincoln's Inn Fields.

We would like to express our gratitude to Ian Hart, PhD, former Professor of Tumour Biology and Deputy Director of Barts Cancer Institute, Queen Mary, University of London, and to Eugene Herman, PhD, Research Pharmacologist, Food and Drug Administration, Division of Applied Pharmacology Research, Silver Spring, Maryland, USA, for contributions and memories.

\section{Websites}

Metastasis Research Society: www.metastasis-research.org Clinical \& Experimental Metastasis: http://www.springer. com/biomed/cancer/journal/10585

MRS Brisbane 2012 (Sept 2-5): http://www.metastasisresearch2012.org 